\title{
$(B, N)$-Pairs; Parabolic, Levi, and Reductive Subgroups; Centralisers of Semi-Simple Elements
}

\section{1 $(B, N)$-Pairs}

We review properties of reductive groups related to existence of a $(B, N)$-pair. For an abstract group, having a $(B, N)$-pair is a very strong condition; many of the theorems we will give for reductive groups follow from this single property.

Definition 3.1.1 We say that two subgroups $B$ and $N$ of a group $G$ form a $(B, N)$-pair (also called a Tits system) for $G$ if:

(i) $B$ and $N$ generate $G$ and $T:=B \cap N$ is normal in $N$.

(ii) The group $W:=N / T$ is generated by a set $S$ of involutions such that:

(a) For $s \in S, w \in W$ we have $B s B . B w B \subset B w B \cup B s w B$.

(b) For $s \in S$, we have $s B s \nsubseteq B$.

The group $W$ is called the Weyl group of the $(B, N)$-pair. Note that we write elements of $W$ - instead of representatives of them in $N$ - in expressions representing subsets of $G$ when these expressions do not depend upon the chosen representative.

We will see in 3.1.3(v) that under the assumptions of 3.1.1 we have $S=\{w \in$ $W-\{1\} \mid B \cup B w B$ is a group $\}$, thus $S$ is determined by $(B, N)$.

Proposition 3.1.2 If $\mathbf{G}$ is a connected reductive group and $\mathbf{T} \subset \mathbf{B}$ is a pair of a maximal torus and a Borel subgroup, then $\left(\mathbf{B}, N_{\mathbf{G}}(\mathbf{T})\right)$ is a $(B, N)$-pair for $\mathbf{G}$.

Proof We show first that $\mathbf{B} \cap N_{\mathbf{G}}(\mathbf{T})=\mathbf{T}$. By 1.3.1(iii) we have $N_{\mathbf{B}}(\mathbf{T})=$ $C_{\mathbf{B}}(\mathbf{T}) \subset C_{\mathbf{G}}(\mathbf{T})=\mathbf{T}$ (see 2.3.1(iii)). By definition $\mathbf{T}$ is normal in $N_{\mathbf{G}}(\mathbf{T})$. To prove (i) it remains to show that $\mathbf{B}$ and $N_{\mathbf{G}}(\mathbf{T})$ generate $\mathbf{G}$. Let $\Phi^{+}$be the positive subsystem defined by B. By 2.3.1(vi), B contains all the $\mathbf{U}_{\alpha}\left(\alpha \in \Phi^{+}\right)$. Since $s_{\alpha}$ conjugates $\mathbf{U}_{\alpha}$ to $\mathbf{U}_{s_{\alpha}(\alpha)}=\mathbf{U}_{-\alpha}$, the group generated by $\mathbf{B}$ and $N_{\mathbf{G}}(\mathbf{T})$ contains $\mathbf{T}$ and all the $\mathbf{U}_{\alpha}(\alpha \in \Phi)$, thus by 2.3.1(v) this group is equal to $\mathbf{G}$. 
If $\Pi$ is the basis defined by the ordering $\Phi^{+}$, (ii) is obtained by taking for $S$ the $\left\{s_{\alpha} \mid \alpha \in \Pi\right\}$.

(ii)(b) reflects that ${ }^{s_{\alpha}} \mathbf{U}_{\alpha}=\mathbf{U}_{-\alpha}$ is not in $\mathbf{B}$.

It remains to show (ii)(a). Let $s=s_{\alpha}$, and write $\mathbf{B}=\mathbf{T} \prod_{\beta \in \Phi^{+}} \mathbf{U}_{\beta}$. As $s$ normalises $\mathbf{T}$, as ${ }^{s} \mathbf{U}_{\beta}=\mathbf{U}_{s_{\alpha}(\beta)}$ and as $s_{\alpha}(\beta) \in \Phi^{+}$if $\beta \in \Phi^{+}-\{\alpha\}$, we get $\mathbf{B} s \mathbf{B} w \mathbf{B}=\mathbf{B} s \mathbf{U}_{\alpha} w \mathbf{B}$. If $w^{-1}(\alpha) \in \Phi^{+}$the right hand side is equal to $\mathbf{B} s w \mathbf{B}$. Otherwise we write it as $\mathbf{B} s \mathbf{U}_{\alpha} s s w \mathbf{B}$ where this time $(s w)^{-1}(\alpha) \in \Phi^{+}$. Let $\mathbf{B}_{\alpha}$ be the image by $\phi_{\alpha}$ (see 2.3.1(ii)) of the Borel subgroup of $\mathbf{S L}_{2}$ of upper triangular matrices. If $c \neq 0$ we have in $\mathbf{S L}_{2}$ :

$$
\left(\begin{array}{ll}
a & b \\
c & d
\end{array}\right)=\left(\begin{array}{cc}
-1 / c & -a \\
0 & -c
\end{array}\right)\left(\begin{array}{cc}
0 & 1 \\
-1 & 0
\end{array}\right)\left(\begin{array}{cc}
1 & d / c \\
0 & 1
\end{array}\right)
$$

which taking images shows that $s \mathbf{U}_{\alpha} s \subset \operatorname{Im} \phi_{\alpha}=\mathbf{B}_{\alpha} \cup \mathbf{B}_{\alpha} s \mathbf{U}_{\alpha}$, whence $\mathbf{B} s \mathbf{U}_{\alpha} s s w \mathbf{B} \subset \mathbf{B} s \mathbf{U}_{\alpha} s w \mathbf{B} \cup \mathbf{B} s w \mathbf{B}$ where the first term in the right-hand side is $\mathbf{B} w \mathbf{B}$ since $(s w)^{-1}(\alpha) \in \Phi^{+}$.

Theorem 3.1.3 If $G$ has a $(B, N)$-pair, then

(i) $G=\bigsqcup_{w \in W} B w B$ (Bruhat decomposition).

(ii) $(W, S)$ is a Coxeter group.

(iii) Condition (ii)(a) of 3.1.1 can be refined to

$$
B s B . B w B= \begin{cases}B s w B & \text { if } l(s w)=l(w)+1, \\ B s w B \cup B w B & \text { otherwise. }\end{cases}
$$

(iv) For any $t \in N(w)$ (see 2.1.2(ii)), we have $B t B \subset B w^{-1} B w B$.

(v) $S=\{w \in W-\{1\} \mid B \cup B w B$ is a group $\}$.

(vi) We have $N_{G}(B)=B$.

Proof Let us show (i). As $B$ and $N$ generate $G$, we have $G=\cup_{i}(B N B)^{i}$. Since $B N B=B W B$ we will get $G=B W B$ if we show that $B W B W B=B W B$. For this it is enough to show that $B w B W B \subset B W B$ for $w \in W$; writing $w=s_{1} \ldots s_{n}$ with $s_{i} \in S$, since $B w B \subset B s_{1} B \ldots B s_{n} B$ it is enough to show $B s B W B \subset B W B$ for $s \in S$; but this results from 3.1.1(ii)(a). It remains to show that $B w B \neq B w^{\prime} B$ if $w \neq w^{\prime}$. We show this by induction on $\inf \left(l(w), l\left(w^{\prime}\right)\right)$, where $l$ is the length with respect to $S$; assume for instance that $l(w) \leq l\left(w^{\prime}\right)$. The start of the induction is $l(w)=0$ and the result comes from $w^{\prime} \notin B$. Otherwise, taking $s \in S$ such that $l(s w)<l(w)$, by induction $B s w B$ is equal neither to $B w^{\prime} B$ nor to $B s w^{\prime} B$ thus $B s w B \cap B s B . B w^{\prime} B=\emptyset$; as $B s w B \subset B s B . B w B$ it follows that $B w B \neq B w^{\prime} B$.

For (ii), we use 2.2.9 with $D_{s}=\{w \in W \mid B s B w B=B s w B\}$ (note that if $w \notin D_{s}$ then $\left.B s B w B=B s w B \amalg B w B\right)$. Clearly $D_{s} \ni 1$. 
If $w, s w \in D_{s}$, then from $B s B w B=B s w B$ and $B s B s w B=B w B$ we get $B s B s B w B=B w B$ which is a contradiction since multiplying on the right by $B w B$ the equality $B s B s B=B s B \amalg B$ (since $s B s \not \subset B$ by 3.1.1(ii)(b)), we get $B s B s B w B=B s w B \amalg B w B$.

It remains to show for (ii) that $w \in D_{s}, w s^{\prime} \notin D_{s}$ implies $w s^{\prime}=s w$. The assumption $w s^{\prime} \notin D_{s}$ implies $B s B w s^{\prime} B=B s w s^{\prime} B \amalg B w s^{\prime} B$; in particular $B s B w s^{\prime}$ meets $B w s^{\prime} B$; multiplying on the right by $s^{\prime} B$ it follows that $B s B w B$ meets $B w s^{\prime} B s^{\prime} B \subset\left(B w B \amalg B w s^{\prime} B\right)$ (this last inclusion follows from 3.1.1(ii)(a) reversed, which is obtained by taking inverses). Thus $B s w B=B s B w B$ (since $w \in D_{s}$ ) is equal to $B w s^{\prime} B$, or to $B w B$. The latter cannot happen since $w \neq s w$, thus $s w=w s^{\prime}$ as was to be shown. We have also shown (iii) by the property of $D_{s}$ given in the last sentence of 2.2.9.

Let us show (iv). If $w=s_{1} \ldots s_{k}$ is a reduced expression, for all $i$ we can write by (iii) $B w B=B s_{1} \ldots s_{i-1} B s_{i} B s_{i+1} \ldots s_{k} B$ and similarly for $B w^{-1} B$ whence

$$
\begin{aligned}
B w^{-1} B w B & =B s_{k} \ldots s_{i+1} B s_{i} B s_{i-1} \ldots s_{1} B s_{1} \ldots s_{i-1} B s_{i} B s_{i+1} \ldots s_{k} B \\
& \supset B s_{k} \ldots s_{i+1} B s_{i} B s_{i} B s_{i+1} \ldots s_{k} B \\
& \supset B s_{k} \ldots s_{i+1} B s_{i} B s_{i+1} \ldots s_{k} B \\
& \supset B s_{k} \ldots s_{i+1} s_{i} s_{i+1} \ldots s_{k} B
\end{aligned}
$$

whence the result.

(v) follows immediately from (iv), which implies that $B \cup B w B$ can be a group only if $|N(w)| \leq 1$, and from (iii) which implies that $B \cup B s B$ is a group.

(vi) also follows from (iv). For $g \in B w B$ we have ${ }^{g} B=B \Leftrightarrow{ }^{w} B=B \Leftrightarrow$ $B w B w^{-1} B=B$ which by (iv) happens only for $w=1$.

In a group $G$ with a $(B, N)$-pair, we call Borel subgroups the conjugates of $B$ and maximal tori the conjugates of $T$; this fits the terminology for algebraic groups.

Corollary 3.1.4 In a group $G$ with a $(B, N)$-pair, every pair of Borel subgroups is conjugate to a pair of the form $\left(B,{ }^{w} B\right)$ with $w \in W$; the intersection of two Borel subgroups contains a maximal torus.

Proof Up to conjugacy, we may assume the given pair of Borel subgroups of the form $\left(B,{ }^{g} B\right)$. By the Bruhat decomposition we may write $g=b w b^{\prime}$ where $b, b^{\prime} \in B$; thus the pair is equal to $\left(B,{ }^{b w} B\right)$, which is conjugate to $\left(B,{ }^{w} B\right)$. Since $B$ and ${ }^{w} B$ both contain $T$, the intersection of every conjugate pair also contains a maximal torus.

Example 3.1.5 For $m$ a matrix in $\mathbf{G L}_{n}$, let $m_{i, j}$ be the submatrix on the last lines $i, \ldots, n$ and first columns $1, \ldots, j$. Let $w$ be a permutation matrix; then 
$m \in \mathbf{B} w \mathbf{B}$, where $\mathbf{B}$ is the Borel subgroup of upper triangular matrices, if and only if the matrices $m_{i, j}$ and $w_{i, j}$ have same rank for all $i, j$. Indeed,

- The ranks of $m_{i, j}$ are invariant by left or right multiplication of $m$ by an upper triangular matrix.

- A permutation matrix $w$ for the permutation $\sigma$ is characterised by the ranks of $w_{i, j}$, given by $|\{k \leq j \mid \sigma(k) \geq i\}|$.

If $\left\{F_{i}^{\prime}\right\}$ and $\left\{F_{i}^{\prime \prime}\right\}$ are two complete flags whose stabilisers are the Borel subgroups $\mathbf{B}^{\prime}$ and $\mathbf{B}^{\prime \prime}$, then the permutation matrix $w$ such that $\left(\mathbf{B}^{\prime}, \mathbf{B}^{\prime \prime}\right)$ is conjugate to $\left(\mathbf{B},{ }^{w} \mathbf{B}\right)$ (the relative position of the two flags) is characterised by $\operatorname{rank} w_{i, j}=\operatorname{dim} \frac{F_{i}^{\prime} \cap F_{j}^{\prime \prime}}{\left(F_{i-1}^{\prime} \cap F_{j}^{\prime \prime}\right)+\left(F_{i}^{\prime} \cap F_{j-1}^{\prime \prime}\right)}$.

\subsection{Parabolic Subgroups of Coxeter Groups and of $(B, N)$-Pairs}

Lemma 3.2.1 Let $(W, S)$ be a Coxeter system, let I be a subset of $S$, and let $W_{I}$ be the subgroup of $W$ generated by $I$, called a standard parabolic subgroup of $W$. Then $\left(W_{I}, I\right)$ is a Coxeter system.

An element $w \in W$ is said to be reduced-I if it satisfies one of the equivalent conditions:

(i) For any $v \in W_{I}$, we have $l(w v)=l(w)+l(v)$.

(ii) For any $s \in I$, we have $l(w s)>l(w)$.

(iii) $w$ has minimal length in the coset $w W_{I}$.

(iv) $N(w) \cap I=\emptyset$.

(v) $N(w) \cap \operatorname{Ref}\left(W_{I}\right)=\emptyset$.

There is a unique reduced-I element in $w W_{I}$.

By exchanging left and right we have the notion of $I$-reduced element which satisfies the mirror properties. A subgroup of $W$ conjugate to a standard parabolic subgroup is called a parabolic subgroup.

Proof A reduced expression in $W_{I}$ is reduced in $W$ by the exchange condition and then satisfies the exchange condition in $W_{I}$, thus $\left(W_{I}, I\right)$ is a Coxeter system.

(iii) $\Rightarrow$ (ii) since (iii) implies $l(w s) \geq l(w)$ when $s \in I$. Let us show that "not (iii)" $\Rightarrow$ "not (ii)". If $w^{\prime}$ does not have minimal length in $w^{\prime} W_{I}$, then $w^{\prime}=w v$ with $v \in W_{I}$ and $l(w)<l\left(w^{\prime}\right)$; adding one by one the terms of a reduced expression for $v$ to $w$ and applying at each stage the exchange condition, we find that $w^{\prime}$ has a reduced expression of the shape $\hat{w} \hat{v}$ where $\hat{w}$ (resp. $\hat{v}$ ) denotes 
a subsequence of the chosen reduced expression. As $l(\hat{w}) \leq l(w)<l\left(w^{\prime}\right)$, we have $l(\hat{v})>0$, thus $w^{\prime}$ has a reduced expression ending by an element of $I$, thus $w^{\prime}$ does not satisfy (ii).

(i) $\Rightarrow$ (iii) is clear. Let us show "not (i)" $\Rightarrow$ "not (iii)". If $l(w v)<l(w)+l(v)$ then a reduced expression for $w v$ has the shape $\hat{w} \hat{v}$ where $l(\hat{w})<l(w)$. Then $\hat{w} \in w W_{I}$ and has a length smaller than that of $w$.

By 2.1.6(ii) property (ii) is equivalent to (iv).

It is clear that (v) implies (iv), and (i) applied to $v \in \operatorname{Ref}(W)$ implies (v) by 2.1.6(ii).

Finally, an element satisfying (i) is clearly unique in $w W_{I}$.

Lemma 3.2.2 Let I and J be two subsets of S. An element $w \in W$ is I-reduced$J$ if it satisfies one of the equivalent properties:

(i) $w$ is both I-reduced and reduced-J.

(ii) $w$ has minimal length in $W_{I} w W_{J}$.

(iii) Every element of $W_{I} w W_{J}$ can be written uniquely $x w y$ with $x \in W_{I}, y \in W_{J}$, $l(x)+l(w)+l(y)=l(x w y)$ and $x w$ is reduced $-J$.

(iii) implies that in a double coset in $W_{I} \backslash W / W_{J}$ there is a unique $I$-reduced- $J$ element, which has minimal length; by symmetry we can replace in condition (iii) the assumption that $x w$ is reduced- $J$ by the assumption that $w y$ is $I$-reduced.

Proof We first show that two elements $w, w^{\prime}$ in the same double coset and satisfying (i) have the same length. Write $w^{\prime}=x w y$ with $x \in W_{I}$ and $y \in W_{J}$; then $w^{\prime} y^{-1}=x w$ and $x^{-1} w^{\prime}=w y$; by the defining properties of $I$-reduced and reduced- $J$ and using $l\left(y^{-1}\right)=l(y), l\left(x^{-1}\right)=l(x)$ we get $l\left(w^{\prime}\right)+l(y)=l(x)+l(w)$ and $l(x)+l\left(w^{\prime}\right)=l(w)+l(y)$, whence $l(x)=l(y)$ and $l(w)=l\left(w^{\prime}\right)$. As clearly (ii) $\Rightarrow$ (i) this common length must be the minimal length, thus (i) $\Leftrightarrow(i i)$.

We now show (ii) $\Rightarrow$ (iii). Assume $w$ satisfies (ii); write an element $v \in$ $W_{I} w W_{J}$ as $x w y$ with $x \in W_{I}, y \in W_{J}$ and $x$ of minimal possible length. By the exchange lemma a reduced expression for $x w y$ is of the form $\hat{x} \hat{w} \hat{y}$ where $\hat{x}$ (resp. $\hat{w}, \hat{y}$ ) is a subsequence of a reduced expression for $x$ (resp. $w, y$ ). Necessarily $\hat{w}=w$ otherwise $w$ would not be of minimal length in its double coset. Then the minimal length assumption on $x$ implies $\hat{x}=x$, whence $\hat{y}=y$, thus $l(x)+l(w)+l(y)=l(x w y)$. The element $x w$ is reduced- $J$ otherwise we can write $x w=v^{\prime} y^{\prime}$ where $v^{\prime} \in W_{I} w W_{J}, y^{\prime} \in W_{J}-\{1\}$ and $l\left(v^{\prime}\right)+l\left(y^{\prime}\right)=l(x w)$. Using what we just proved on $w$ we can write $v^{\prime}=x^{\prime \prime} w y^{\prime \prime}$ with $l\left(x^{\prime \prime}\right)+l(w)+l\left(y^{\prime \prime}\right)+l\left(y^{\prime}\right)=l(x)+l(w)$ which implies $l\left(x^{\prime \prime}\right)<l(x)$, contradicting the minimality of $l(x)$. Finally the decomposition $x w y$ is unique since $x w$ is the unique $J$-reduced element in its coset.

Finally, (iii) $\Rightarrow$ (ii) is clear. 
Note that not every decomposition $x w y$ where $w$ is $I$-reduced- $J$ satisfies (iii); consider for instance the case $w=y=1, I=J$ and $x$ the longest element of $W_{I}$; thus the situation is not as good as in the $I$-reduced case.

In a group with a $(B, N)$-pair, we use the term parabolic subgroups for the subgroups containing a Borel subgroup.

\section{Proposition 3.2.3 Let $G$ be a group with a $(B, N)$-pair. Then}

(i) The (parabolic) subgroups containing $B$ are the $P_{I}=B W_{I} B$ for some $I \subset S$.

(ii) Given two parabolic subgroups $P_{I}$ and $P_{J}$, we have a relative Bruhat decomposition $G=\coprod_{w} P_{I} w P_{J}$ where $w$ runs over the I-reduced-J elements. It follows a natural bijection $P_{I} \backslash G / P_{J} \stackrel{\sim}{\rightarrow} W_{I} \backslash W / W_{J}$.

Proof Let us show (i). Let $P$ be a subgroup containing $B$ and let $w \in W$ be such that $B w B \subset P$. Since $P$ is a group we get $B w^{-1} B w B \subset P$, thus by 3.1.3(iv) we get $B t B \subset P$ for any $t \in N(w)$. If $w=s_{1} \ldots s_{k}$ is a reduced expression we get in particular $B s_{k} B \subset P$, thus $s_{1} \ldots s_{k-1} \in P$ and by induction for each $i$ we have $s_{i} \in P$. It follows that $P=B W_{I} B$ where $I$ is the union of the elements of $S$ appearing in any reduced expression of any $w$ such that $B w B \subset P$. Conversely, for any $I \subset S$, using 3.1.1(ii)(a) we see that $B W_{I} B$ is a group.

Let us show (ii). For any $w \in W$ we have $P_{I} w P_{J}=B W_{I} B w B W_{J} B=$ $B W_{I} w W_{J} B$, the last equality by repeated application of 3.1.1(ii)(a) and of its right counterpart. Since, by Lemma 3.2.2 we can take $I$-reduced- $J$ elements as representatives of the double cosets we see that the first assertion of (ii) is just the Bruhat decomposition. Conversely, any coset $P_{I} g P_{J}$ is of the form $P_{I} w P_{J}$ if $g \in B w B$ whence the last assertion of (ii).

Remark 3.2.4 Using 3.2.3 we see that in the definition 1.3.5 of a parabolic subgroup the word "closed" can be omitted. Indeed a reductive group has a $(B, N)$ pair, hence by 3.2.3 a subgroup containing a Borel subgroup is conjugate to some $\mathbf{B} W_{I} \mathbf{B}$, hence it is closed. In general, if $\mathbf{G}$ is a connected group and $\mathbf{P}$ is a subgroup containing a Borel subgroup, then $\mathbf{P} / R_{u}(\mathbf{G})$ contains a Borel subgroup of the reductive group $\mathbf{G} / R_{u}(\mathbf{G})$ hence it is closed, thus $\mathbf{P}$ is closed by continuity of the quotient morphism.

Example 3.2.5 In $\mathbf{G L}_{n}$, the parabolic subgroup $\mathbf{P}_{J}$ for $J \subset S$ containing the Borel subgroup of upper triangular matrices is the subgroup of upper blocktriangular matrices where the blocks correspond to maximal intervals $[i, k]$ in $[1, n]$ such that $s_{i}, \ldots, s_{k-1} \in J$.

Example 3.2.6 For the symplectic group $\mathbf{S p}_{2 n}$, as the stabiliser $\mathbf{B}$ of any complete isotropic flag $V_{1} \subset \cdots \subset V_{n}$ in $\mathbf{S p}_{2 n}$ is a Borel subgroup, the stabiliser of any subflag is a parabolic subgroup. We thus get $2^{n}$ distinct parabolic subgroups containing $\mathbf{B}$. Since there are also $2^{n}$ subsets of $S$, they are the only parabolic 
subgroups containing B. As any isotropic flag may be completed to a complete one, we get the result that in general parabolic subgroups are the stabilisers of (complete or not) isotropic flags.

Lemma 3.2.7 (unicity in Bruhat decomposition) Let $\mathbf{G}$ be a connected reductive group and $\mathbf{B}=\mathbf{U} \rtimes \mathbf{T}$ be a decomposition of a Borel subgroup $\mathbf{B}$ as in 1.3.1(ii), where $\mathbf{U}=R_{u}(\mathbf{B})$. Then $\mathbf{B} w \mathbf{B}$ has a direct product decomposition $\mathbf{U} \times \mathbf{T} w \times \mathbf{U}_{w}$ where $\mathbf{U}_{w}:=\prod_{\left\{\alpha \in \Phi^{+} \mid w(\alpha)<0\right\}} \mathbf{U}_{\alpha}$.

Proof Notice first that $\mathbf{U}_{w}$ is a group; since if in 2.3.1(vii) $\alpha$ and $\beta$ are sent to negative roots by $w$, then the same holds for $\lambda \alpha+\mu \beta$. We have $\mathbf{U}=\mathbf{U}^{\prime} \mathbf{U}_{w}$ where $\mathbf{U}^{\prime}=\prod_{\left\{\alpha \in \Phi^{+} \mid w(\alpha)>0\right\}} \mathbf{U}_{\alpha}$ thus ${ }^{w} \mathbf{U}^{\prime} \subset \mathbf{U}$; thus $\mathbf{B} w \mathbf{B}=\mathbf{U T} w \mathbf{U}^{\prime} \mathbf{U}_{w}=$ $\mathbf{U T} w \mathbf{U}_{w}$. It remains to be shown that the decomposition is unique; that is, if $u \mathbf{T} w u^{\prime}=\mathbf{T} w$ with $u \in \mathbf{U}, u^{\prime} \in \mathbf{U}_{w}$ then $u=u^{\prime}=1$. The condition implies ${ }^{w} u^{\prime} \in \mathbf{B}$. But ${ }^{w} \mathbf{U}_{w} \cap \mathbf{B}=1$ since all $\mathbf{U}_{\alpha}$ in ${ }^{w} \mathbf{U}_{w}$ are for negative $\alpha$. Thus $u^{\prime}=1$, whence $u=1$.

The next proposition says that the decomposition of $\mathbf{G}$ in Bruhat cells $\mathbf{B} w \mathbf{B}$ is a stratification (the closure of a stratum is a union of strata).

Proposition 3.2.8 Let $\mathbf{G}$ be a connected reductive group and $\mathbf{B}=\mathbf{U} \rtimes \mathbf{T}$ be a decomposition of a Borel subgroup $\mathbf{B}$ as in 3.2.7. Then the Zariski closure of $\mathbf{B} w \mathbf{B}$ in $\mathbf{G}$ is given by $\overline{\mathbf{B} w \mathbf{B}}=\coprod_{v \leq w} \mathbf{B} v \mathbf{B}$, where $\leq$ is the Bruhat-Chevalley order on $w$, given by $v \leq w$ if a reduced expression of $v$ is a subsequence of a reduced expression of $w$.

Reference See Chevalley (1994, Proposition 6).

\subsection{Closed Subsets of a Crystallographic Root System}

In this section, $\Phi$ will be a reduced crystallographic root system in the $\mathbb{Q}$-vector space $V$, and $\Pi$ will be a basis of $\Phi$; we denote by $\Phi^{+}$the corresponding positive subsystem and by $(W, S)$ the corresponding Coxeter system, where

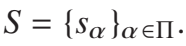

Definition 3.3.1 A subset $\Psi \subset \Phi$ is:

(i) closed if $\alpha, \beta \in \Psi, \alpha+\beta \in \Phi \Rightarrow \alpha+\beta \in \Psi$.

(ii) symmetric if $\Psi=-\Psi$.

The intersection of two closed subsets is clearly closed.

Lemma 3.3.2 The reduced crystallographic root systems of rank 2 are $A_{1} \times A_{1}$, $A_{2}, C_{2}=B_{2}$ and $G_{2}$. 
Here is a picture of their positive roots:

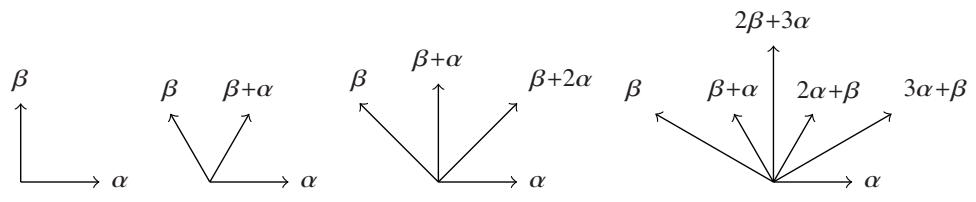

Proof Let $\Phi$ be crystallographic of rank 2 with Weyl group $W$. Let $\Pi=\{\alpha, \beta\}$. Choosing a $W$-invariant scalar product (.,.) as in 2.2.3, we have $\alpha^{\vee}(\beta) \beta^{\vee}(\alpha)=$ $4 \frac{(\alpha, \beta)^{2}}{(\alpha, \alpha)(\beta, \beta)}=4 \cos ^{2} \theta$ where $\theta$ is the angle between $\alpha$ and $\beta$. Since $\alpha, \beta \in \Pi$ we have $(\alpha, \beta) \leq 0$ thus $\pi / 2 \leq \theta \leq \pi$ and the integrality of $4 \cos ^{2} \theta$ implies that $4 \cos ^{2} \theta \in\{0,1,2,3\}$ thus $\pi-\theta \in\{\pi / 2, \pi / 3, \pi / 4, \pi / 6\}$. Except for $A_{1} \times A_{1}$, the ratio of the lengths of $\alpha$ and $\beta$ is implied by the equation $\alpha^{\vee}(\beta) \beta^{\vee}(\alpha)=$ $4 \cos ^{2} \theta$. For instance if $4 \cos ^{2} \theta=2$ the only integral solution, up to exchanging $\alpha$ and $\beta$, is $\alpha^{\vee}(\beta)=-1$ and $\beta^{\vee}(\alpha)=-2$ whence $2(\beta, \beta)=(\alpha, \alpha)$. For $A_{1} \times A_{1}$ the ratio of the lengths is not determined, we have chosen 1 in the picture.

Corollary 3.3.3 For a crystallographic root system $\Phi$ and a positive subsystem $\Phi^{+}$, we have:

(i) If $\alpha, \beta \in \Phi, \alpha \neq-\beta$ and $(\alpha, \beta)<0$, then $\alpha+\beta \in \Phi$.

(ii) If $\alpha, \beta \in \Phi$ and $\alpha+n \beta \in \Phi$ for $n \in \mathbb{N}$, then $\alpha+m \beta \in \Phi$ for all $0 \leq m \leq n$.

(iii) If $\alpha_{1}, \ldots, \alpha_{k} \in \Phi^{+}$and $\alpha=\alpha_{1}+\cdots+\alpha_{k} \in \Phi^{+}$, then if $k>1$ we have $\alpha-\alpha_{i} \in \Phi^{+}$for some $i$.

(iv) If $\Psi \subset \Phi$ is closed, $\alpha, \beta \in \Psi, \alpha \neq-\beta$ and $n \alpha+m \beta \in \Phi$ for some $n, m>0$, then $n \alpha+m \beta \in \Psi$.

Proof For (i), by the argument in the proof of 3.3.2 about possible integral solutions, up to exchanging $\alpha$ and $\beta$ we have $\alpha^{\vee}(\beta)=-1$, whence $\alpha+\beta=$ $s_{\alpha}(\beta) \in \Phi$. For (iii) as $(\alpha, \alpha)>0$ we must have $\left(\alpha, \alpha_{i}\right)>0$ for some $i$ thus by (i) $\alpha-\alpha_{i} \in \Phi$.

For (ii), by (iii) either $\alpha+(n-1) \beta$ or $n \beta$ is in $\Phi$, and since $\Phi$ is reduced, $n \beta \notin \Phi$ if $n \neq 1$, whence the result by induction on $n$.

For (iv) we may assume both $\alpha$ and $\beta$ positive (they are for some order since $\alpha \neq-\beta$ ), and then we apply (iii) and induction on $n+m$.

Corollary 3.3.4 If $\Psi \subset \Phi$ is closed and symmetric, it is a root subsystem.

Proof For $\alpha, \beta \in \Psi$, we have to show that $s_{\alpha}(\beta) \in \Psi$. This is true if $\beta=$ $\pm \alpha$ since $\Psi$ is symmetric. Otherwise, replacing $\alpha$ by $-\alpha$ if necessary we have $s_{\alpha}(\beta)=\beta+n \alpha$ for some $n \in \mathbb{N}^{*}$; then Corollary 3.3.3(iv) gives the result.

Proposition 3.3.5 If $\Psi$ is closed and $\Psi \cap-\Psi=\emptyset$, there exists a positive subsystem $\Phi^{+}$such that $\Psi \subset \Phi^{+}$. 
Proof We first show by induction on $k>0$ that 0 is not the sum of $k$ elements of $\Psi$. This is clear for $k=1$. If $0=\alpha_{1}+\cdots+\alpha_{k}$ then $0<\left(\alpha_{1}, \alpha_{1}\right)=$ $\left(-\alpha_{1}, \alpha_{2}+\cdots+\alpha_{k}\right)$ thus there exists $i \neq 1$ such that $\left(\alpha_{1}, \alpha_{i}\right)<0$. Using $\alpha_{1} \neq-\alpha_{i}$ (since $-\alpha_{i} \notin \Psi$ by assumption) and 3.3.3(i) we get $\alpha_{1}+\alpha_{i} \in \Phi$ thus $\alpha_{1}+\alpha_{i} \in \Psi$, thus the sum is the sum of $k-1$ elements, a contradiction.

We now build by induction on $k$ a sequence $\gamma_{k}$ of elements of $\Psi$ such that $\gamma_{k} \in \Psi$ is the sum of $k$ elements of $\Psi$. We start with $\gamma_{1}$ equal to an arbitrary element of $\Psi$. If there is $\alpha \in \Psi$ such that $\left(\gamma_{k}, \alpha\right)<0$ we set $\gamma_{k+1}=\alpha+\gamma_{k} \in \Psi$. For $i<j$ we have $\gamma_{i} \neq \gamma_{j}$, otherwise $\gamma_{j}-\gamma_{i}$ would be a zero sum of elements of $\Psi$, thus by finiteness the sequence must stop on some $\gamma_{k}$ such that $\left(\gamma_{k}, \alpha\right) \geq 0$ for any $\alpha \in \Psi$. The linear form $\left(\gamma_{k},.\right)$ almost defines an order as in 2.2.4. We need to modify it on $\gamma_{k}^{\perp}$. But $\gamma_{k}^{\perp} \cap \Psi \subset \gamma_{k}^{\perp} \cap \Phi$ satisfies the same assumptions as the proposition and we may iterate the construction on this subspace.

For $I \subset S$ we set $\Pi_{I}:=\left\{\alpha \in \Pi \mid s_{\alpha} \in I\right\}$ and $\Phi_{I}=\Phi \cap \mathbb{Q}_{I}$; it is clearly a root subsystem with basis $\Pi_{I}$, since when decomposed on $\Pi$ a root of $\Phi_{I}$ involves only elements of $\Pi_{I}$.

It is clear that $\Phi_{I}$ is closed and symmetric and that $\Phi^{+}-\Phi_{I}$ and $\Phi^{+} \cup \Phi_{I}$ are closed.

Example 3.3.6 There exist closed and symmetric subsystems which are not of the form $\Phi_{I}$; for instance the long roots in a system $B_{2}$ form a system of type $A_{1} \times A_{1}$, and the long roots in $G_{2}$ form a system of type $A_{2}$. See also 11.2.7.

Lemma 3.3.7 If $s_{\alpha} \in W_{I}$ for $\alpha \in \Phi$, then $\alpha \in \Phi_{I}$.

Proof Elements of $W_{I}$ are the product of some $s_{\beta}$ for $\beta \in \Pi_{I}$, thus they fix $\Pi_{I}^{\perp}$. Thus $s_{\alpha}$ fixes $\Pi_{I}^{\perp}$, which implies that $\alpha \in \mathbb{Q}_{I} \cap \Phi=\Phi_{I}$.

We say that $\Psi$ is a parabolic subset of $\Phi$ if $\Psi$ is closed and $\Psi \cup-\Psi=\Phi$.

\section{Proposition 3.3.8}

(i) A parabolic subset is conjugate to a parabolic subset containing $\Phi^{+}$; such a subset is of the form $\Phi^{+} \cup \Phi_{I}$ for some $I \subset S$.

(ii) A parabolic subset is a set of the form $\{\alpha \mid \lambda(\alpha) \geq 0\}$ for some linear form $\lambda$ on $V$.

Proof For the first part of (i) it is equivalent to show that a parabolic subset $\Psi$ contains some positive subsystem. Choose such a positive subsystem $\Phi^{+}$such that $\left|\Psi \cap \Phi^{+}\right|$is maximal. We show by contradiction that $\Phi^{+} \subset \Psi$. Otherwise let $\Pi$ be the basis of $\Phi$ defining $\Phi^{+}$; there must exist $\alpha \in \Pi, \alpha \notin \Psi$, thus $-\alpha \in \Psi$. Since $\alpha \notin \Psi$ we have $s_{\alpha}\left(\Psi \cap \Phi^{+}\right) \subset \Phi^{+}$; applying $s_{\alpha}$ again we get $\Psi \cap \Phi^{+} \subset s_{\alpha}\left(\Phi^{+}\right)$. But then the positive subsystem $s_{\alpha}\left(\Phi^{+}\right)$contains $-\alpha$ thus satisfies $\left|\Psi \cap s_{\alpha}\left(\Phi^{+}\right)\right|>\left|\Psi \cap \Phi^{+}\right|$, a contradiction. 
We now assume that $\Psi \supset \Phi^{+}$. Let $I=\left\{s_{\alpha} \mid-\alpha \in\{-\Pi \cap \Psi\}\right\}$. Let us show that $\Psi \cap \Phi^{-}=\Phi_{I}^{-}$.

We first show that $\Phi_{I}^{-} \subset \Psi$. Note that by 2.2 .8 applied to the basis $-\Pi_{I}$ of $\Phi_{I}^{-}$any root in $\Phi_{I}^{-}$is a sum of elements of $-\Pi_{I}$. We show by induction on $k$ that a root in $\Phi_{I}^{-}$sum of $k$ roots in $-\Pi_{I}$ is in $\Psi$. It is true by assumption when $k=$ 1 ; in general by 3.3.3(iii) we may write the root as $\alpha+\beta$ where $\alpha \in-\Pi_{I}$ and $\beta \in \Phi_{I}^{-}$sum of $k-1$ roots in $-\Pi_{I}$; by induction $\beta \in \Psi$ and as $\alpha \in \Psi$ and $\Psi$ is closed $\alpha+\beta \in \Psi$.

We finally show the reverse inclusion by induction. Let $\gamma \in \Psi \cap \Phi^{-}$be the sum of $k$ roots of $-\Pi$, and write it $\gamma=\alpha+\beta$ where $\alpha \in-\Pi$ and $\beta \in \Phi$ is the sum of $k-1$ roots in $-\Pi$. As $-\beta \in \Phi^{+} \subset \Psi$ we get $\alpha=\gamma+(-\beta) \in \Psi$ whence $\alpha \in-\Pi \cap \Psi=-\Pi_{I}$. Thus $-\alpha \in \Psi$ whence $\beta=\gamma+(-\alpha) \in \Psi$, and we conclude since by induction $\beta \in \Phi_{I}^{-}$.

Conversely the fact that for any $I \subset S$ the set $\Phi^{+} \cup \Phi_{I}$ is parabolic is a consequence of the proof of (ii) below.

We now show (ii). It is clear that a subset of the form $\{\alpha \mid \lambda(\alpha) \geq 0\}$ is parabolic. It is thus sufficient to show that $\Phi^{+} \cup \Phi_{I}$ is of this form. Take any $x$ such that $\langle x, \alpha\rangle=0$ if $\alpha \in \Phi_{I}$ and $\langle x, \alpha\rangle>0$ if $\alpha \in \Psi-\Phi_{I}$. Such an $x$ exists: the projection of $\Phi^{+}$on $\Phi_{I}^{\perp}$ lies in a half-space, and we may take $x$ in this half-space, orthogonal to the hyperplane which delimits it. It is clear that by construction $x$ has the required properties.

A consequence of 3.3.8 is that the complement of a parabolic subset is closed.

\section{Subgroups of Maximal Rank and Quasi-closed Sets}

In the remainder of this chapter $\mathbf{G}$ is a connected reductive algebraic group, $\mathbf{T}$ is a maximal torus of $\mathbf{G}$, and $\Phi$ is the set of roots of $\mathbf{G}$ relative to $\mathbf{T}$. For $\Psi \subset \Phi$, we set $\mathbf{G}_{\Psi}^{*}:=\left\langle\mathbf{U}_{\alpha} \mid \alpha \in \Psi\right\rangle$ and $\mathbf{G}_{\Psi}:=\left\langle\mathbf{T}, \mathbf{U}_{\alpha} \mid \alpha \in \Psi\right\rangle$. These are closed connected subgroups by 1.1.3 and $\mathbf{G}_{\psi}^{*}$ is a normal subgroup of $\mathbf{G}_{\Psi}$.

Definition 3.3.9 A subset $\Psi \subset \Phi$ is called quasi-closed if $\mathbf{G}_{\Psi}^{*}$ does not contain any $\mathbf{U}_{\alpha}$ with $\alpha \in \Phi-\Psi$.

We get an equivalent definition by replacing $\mathbf{G}_{\Psi}^{*}$ with $\mathbf{G}_{\Psi}$, since $\mathbf{G}_{\Psi} / \mathbf{G}_{\Psi}^{*}$ is a quotient of $\mathbf{T}$ and thus is a torus. Hence any $\mathbf{U}_{\alpha} \subset \mathbf{G}_{\Psi}$ is in the kernel of this quotient, and is thus in $\mathbf{G}_{\Psi}^{*}$.

Proposition 3.3.10 A closed and connected subgroup $\mathbf{H} \subset \mathbf{G}$ containing $\mathbf{T}$ is equal to $\mathbf{G}_{\Psi}$ with $\Psi=\left\{\alpha \in \Phi \mid \mathbf{U}_{a} \subset \mathbf{H}\right\}$; the set $\Psi$ is quasi-closed. 
Proof By 2.3.1(iv) $\mathbf{H}$ is generated by $\mathbf{T}$ and the $\mathbf{U}_{\alpha}$ it contains. The subset $\Psi \subset \Phi$ of those $\alpha$ is quasi-closed by definition.

Let $\Psi, \Psi^{\prime}$ be quasi-closed; it is clear that $\Psi \cap \Psi^{\prime}$ is quasi-closed (since $\mathbf{G} \Psi_{\cap} \cap \Psi^{\prime}$ is a subgroup of both $\mathbf{G}_{\Psi}$ and $\left.\mathbf{G}_{\Psi^{\prime}}\right)$; actually one sees that $\mathbf{G}_{\Psi_{\cap} \Psi^{\prime}}=\left(\mathbf{G}_{\Psi} \cap \mathbf{G}_{\Psi^{\prime}}\right)^{0}$ by applying 2.3.1(iv) to the right-hand side.

Definition 3.3.11 A connected linear algebraic group $\mathbf{P}$ has a Levi decomposition if there is a closed subgroup $\mathbf{L} \subset \mathbf{P}$ such that $\mathbf{P}=R_{u}(\mathbf{P}) \rtimes \mathbf{L}$. The group $\mathbf{L}$ is called a Levi subgroup of $\mathbf{P}$ (or a Levi complement).

A Levi complement is clearly reductive.

Proposition 3.3.12 Let $\Psi \subset \Phi$ be quasi-closed, and let $\Psi_{s}=\{\alpha \in \Psi \mid-\alpha \in$ $\Psi\}$ and $\Psi_{u}=\{\alpha \in \Psi \mid-\alpha \notin \Psi\}$. Then $\Psi_{s}$ and $\Psi_{u}$ are quasi-closed and $\mathbf{G}_{\Psi}$ has a Levi decomposition $\mathbf{G}_{\Psi}=\mathbf{G}_{\Psi_{u}}^{*} \rtimes \mathbf{G}_{\Psi_{s}}$ where $\mathbf{G}_{\Psi_{u}}^{*}=R_{u}\left(\mathbf{G}_{\Psi}\right)$. In particular $\mathbf{G}_{\Psi}$ is reductive if and only if $\Psi$ is symmetric.

Proof We first show that $\Psi_{s}$ is quasi-closed. As the intersection of two quasiclosed sets is quasi-closed, it is enough to show that $-\Psi$ is quasi-closed. This results from the existence of the opposition automorphism of $\mathbf{G}$ which acts by -1 on $X(\mathbf{T})$; see Example 2.4.9.

As a connected group normalised by $\mathbf{T}$ the group $R_{u}\left(\mathbf{G}_{\Psi}\right)$ is - by Theorem 2.3.1(v) - of the form $\mathbf{G}_{\Psi^{\prime}}^{*}$ for a subset $\Psi^{\prime} \subset \Psi$ that we may assume quasiclosed. We have $\Psi^{\prime} \subset \Psi_{u}$, otherwise there is $\alpha \in \Psi_{s} \cap \Psi^{\prime}$, thus $\mathbf{U}_{-\alpha} \subset \mathbf{G}_{\Psi}$ thus normalises $R_{u}\left(\mathbf{G}_{\Psi}\right)$ thus $\left[\mathbf{U}_{-\alpha}, \mathbf{U}_{\alpha}\right] \subset R_{u}\left(\mathbf{G}_{\Psi}\right)$ which is a contradiction since this commutator set contains non-unipotent elements by Theorem 2.3.1(ii).

To show $\Psi^{\prime}=\Psi_{u}$ it is thus enough to show $\Psi-\Psi^{\prime} \subset \Psi_{s}$. If $\alpha \in \Psi-$ $\Psi^{\prime}$, then $\mathbf{U}_{\alpha} \cap R_{u}\left(\mathbf{G}_{\Psi}\right)=1$ since this intersection is normalised by $\mathbf{T}$ thus contains the whole $\mathbf{U}_{\alpha}$ if not trivial. Thus, in the quotient $\mathbf{G}_{\Psi} \rightarrow \mathbf{L}^{\prime}$, where $\mathbf{L}^{\prime}$ is the reductive group $\mathbf{G}_{\Psi} / R_{u}\left(\mathbf{G}_{\Psi}\right)$, the group $\mathbf{U}_{\alpha}$ maps injectively to a root subgroup of $\mathbf{L}^{\prime}$. Let $\mathbf{U}^{\prime}$ be the root subgroup of $\mathbf{L}^{\prime}$ corresponding to the opposed root and $\mathbf{U}^{\prime \prime}$ its preimage. Any element of $\mathbf{U}^{\prime \prime}$ is unipotent since, its image being unipotent, its semi-simple part is in $R_{u}\left(\mathbf{G}_{\Psi}\right)$ so is trivial. Hence $\mathbf{U}^{\prime \prime}$ is a unipotent subgroup normalised by $\mathbf{T}$, so is a product of certain root subgroups and must contain $\mathbf{U}_{-\alpha}$, thus $-\alpha \in \Psi$ and $\alpha \in \Psi_{s}$.

It also follows from the proof that $\mathbf{G}_{\Psi_{s}}$ maps injectively to $\mathbf{L}^{\prime}$, thus $\mathbf{G}_{\Psi_{s}}$ is a Levi complement of $R_{u}\left(\mathbf{G}_{\Psi}\right)$.

Proposition 3.3.13 A closed subset is quasi-closed. 
Proof Let $\Psi \subset \Phi$ be a closed subset and let $\Psi_{s}$ and $\Psi_{u}$ be as in 3.3.12. It is clear that $\Psi_{s}$ is closed. Note that if $\alpha \in \Psi, \beta \in \Psi_{u}$ and $\alpha+\beta \in \Phi$ then $\alpha+\beta \in$ $\Psi_{u}$, otherwise $\alpha+\beta \in \Psi_{s}$ whence $-\alpha-\beta \in \Psi_{s}$ thus $\alpha+(-\alpha-\beta)=-\beta \in \Psi$ which contradicts $\beta \in \Psi_{u}$. In particular $\Psi_{u}$ is closed. By 3.3.5 there exists a positive subsystem such that $\Psi_{u} \subset \Phi^{+}$.

Lemma 3.3.14 If $\Psi$ is a closed subset of a positive subsystem $\Phi^{+}$of $\Phi$, then $\Psi$ is quasi-closed and $\mathbf{G}_{\Psi}^{*}=\prod_{\alpha \in \Psi} \mathbf{U}_{\alpha}$ where the product is taken in an arbitrary order.

In the situation of the lemma we will write $\mathbf{U}_{\Psi}$ for $\mathbf{G}_{\Psi}^{*}$.

Proof By 2.3.1(vii) and 3.3.3(iv) $\prod_{\alpha \in \Psi} \mathbf{U}_{\alpha}$ is a group, thus equal to $\mathbf{G}_{\Psi}^{*}$.

We deduce that $\Psi_{u}$ is quasi-closed. In addition, if $\alpha \in \Psi_{s}, \beta \in \Psi_{u}$ and $\alpha+\beta \in \Phi$, using the fact that $\alpha+\beta \in \Psi_{u}$ and 3.3.3(iv), we get $n \alpha+m \beta \in \Psi_{u}$ for $n, m \geq 1$ such that $n \alpha+m \beta \in \Phi$. Thus $\mathbf{G}_{\Psi_{u}}^{*}$ is normalised by $\mathbf{G}_{\Psi_{s}}$.

Since $\Psi_{s}$ is closed and symmetric, it is a root subsystem by 3.3.4. Let $\Pi_{s}$ be its basis corresponding to the positive subsystem $\Psi_{s} \cap \Phi^{+}$. Note that $\mathbf{G}_{\Psi_{s}}$ is already generated by $\mathbf{T}$ and $\mathbf{U}_{\alpha}$ such that $\alpha \in \pm \Pi_{s}$; indeed $\left\langle\mathbf{U}_{\alpha}, \mathbf{U}_{-\alpha}\right\rangle$ contains a representative of $s_{\alpha}$ by 2.3.1(iii), thus $\mathbf{G}_{\Psi_{s}}$ contains $W_{\Psi_{s}}$, and every root of $\Psi_{s}$ is in the orbit of $\Pi_{s}$ by 2.2.8, whence the result by the remark above 2.3.3. We show now that $\mathbf{G}_{\Psi_{s}}=\mathbf{U}_{\Psi_{s}^{+}} W_{\Psi_{s}} \mathbf{T} \mathbf{U}_{\Psi_{s}^{+}}$. For that it is enough to show that the right-hand side is a group. Since it is stable by left translation by $\mathbf{T}$ and by any $\mathbf{U}_{\alpha}$ for $\alpha \in \Psi_{s}^{+}$it is enough to see it is stable by left translation by $\mathbf{U}_{-\alpha}$ for $\alpha \in \Pi_{s}$. Decomposing $\mathbf{U}_{\Psi_{s}^{+}}=\mathbf{U}_{\Psi_{s}^{+}-\{\alpha\}} \mathbf{U}_{\alpha}$, and using that by 2.3.1(vii) $\mathbf{U}_{-\alpha}$ normalises $\mathbf{U}_{\Psi_{s}^{+}-\{\alpha\}}$ since $\alpha$ is simple, it is enough to see that $\mathbf{U}_{\alpha} W_{\Psi_{s}} \mathbf{T} \mathbf{U}_{\Psi_{s}^{+}}$is stable by left translation by $\mathbf{U}_{-\alpha}$. The Bruhat decomposition $\left\langle\mathbf{T}, \mathbf{U}_{\alpha}, \mathbf{U}_{-\alpha}\right\rangle=$ $\mathbf{U}_{\alpha} \mathbf{T} \cup \mathbf{U}_{\alpha} s_{\alpha} \mathbf{T} \mathbf{U}_{\alpha}$ shows that $\mathbf{U}_{-\alpha} \mathbf{U}_{\alpha} \subset \mathbf{U}_{\alpha} \mathbf{T} \cup \mathbf{U}_{\alpha} s_{\alpha} \mathbf{T} \mathbf{U}_{\alpha}$. We just need to consider the second term

$$
\mathbf{U}_{\alpha} s_{\alpha} \mathbf{T} \mathbf{U}_{\alpha} W_{\Psi_{s}} \mathbf{T} \mathbf{U}_{\Psi_{s}^{+}}=\bigcup_{w \in \Psi_{s}} \mathbf{U}_{\alpha} s_{\alpha} \mathbf{U}_{\alpha} w \mathbf{T} \mathbf{U}_{\Psi_{s}^{+}}
$$

If $w^{-1}(\alpha) \in \Psi^{+}$, then $\mathbf{U}_{\alpha} w \mathbf{T}=w \mathbf{T} \mathbf{U}_{w^{-1}(\alpha)}$ and the term has the right form. Otherwise, letting $\beta=-w^{-1}(\alpha) \in \Psi_{s}^{+}$we get

$$
\begin{aligned}
\mathbf{U}_{\alpha} s_{\alpha} w \mathbf{T} \mathbf{U}_{w^{-1}(\alpha)} \mathbf{U}_{\Psi_{s}^{+}} & =\mathbf{U}_{\alpha} s_{\alpha} w \mathbf{T} \mathbf{U}_{-\beta} \mathbf{U}_{\beta} \mathbf{U}_{\Psi_{s}^{+}-\{\beta\}} \\
& \subset \mathbf{U}_{\alpha} s_{\alpha} w \mathbf{T}\left(\mathbf{U}_{\beta} \cup \mathbf{U}_{\beta} s_{\beta} \mathbf{U}_{\beta}\right) \mathbf{U}_{\Psi_{s}^{+}-\{\beta\}} \\
& =\mathbf{U}_{\alpha} s_{\alpha} w \mathbf{T} \mathbf{U}_{\Psi_{s}^{+}} \cup \mathbf{U}_{\alpha} s_{\alpha} w \mathbf{T} \mathbf{U}_{\beta} s_{\beta} \mathbf{U}_{\Psi_{s}^{+}} .
\end{aligned}
$$

We just need to consider the rightmost term. Since $s_{\alpha} w \mathbf{U}_{\beta} \mathbf{T}=s_{\alpha} \mathbf{U}_{-\alpha} w \mathbf{T}=$ $\mathbf{U}_{\alpha} s_{\alpha} w \mathbf{T}$ we get the result. 
Let us now show that $\Psi_{s}$ is quasi-closed. Let $\gamma$ be such that $\mathbf{U}_{\gamma} \subset \mathbf{G}_{\Psi_{s}}$; since $\Psi_{s}=-\Psi_{s}$, we may assume $\gamma \in \Phi^{+}$, thus $\mathbf{U}_{\gamma} \subset \mathbf{B}$. As each term $\mathbf{U}_{\Psi_{s}^{+}} w \mathbf{T} \mathbf{U}_{\Psi_{s}^{+}}$is in a unique Bruhat cell of $\mathbf{G}$, we must have $\mathbf{U}_{\gamma} \subset \mathbf{T} \mathbf{U}_{\Psi_{s}^{+}}$. By Lemma 3.3.14 $\Psi_{s}^{+}$ is quasi-closed, thus $\gamma \in \Psi_{s}^{+}$.

We have seen that $\mathbf{G}_{\Psi}$ has a semi-direct product decomposition $\mathbf{G}_{\Psi_{u}}^{*} \rtimes \mathbf{G}_{\Psi_{s}}$. It follows that $\Psi$ is quasi-closed since if $\alpha \notin \Psi_{u}$ and $\mathbf{U}_{\alpha} \subset \mathbf{G}_{\Psi}$ then $\mathbf{U}_{\alpha}$ maps isomorphically to the quotient $\mathbf{G}_{\Psi_{s}}$ thus $\alpha \in \Psi_{s}$.

Conversely any quasi-closed subset of $\Phi$ is closed apart from some exceptions in characteristics 2 and 3; see Borel and Tits $(1965,3.8)$. This can be shown by proving that in other characteristics the group $\left\langle\mathbf{U}_{\alpha}, \mathbf{U}_{\beta}\right\rangle$ contains all $\mathbf{U}_{n \alpha+m \beta}$ for $n, m \in \mathbb{N}$ such that $n \alpha+m \beta \in \Phi$, using the explicit values of the coefficients in the proof of 2.3.1(vii). For a combinatorial description of quasi-closed subsets in these characteristics, see Malle and Testerman (2011, Corollary 13.7).

\subsection{Parabolic Subgroups and Levi Subgroups}

Proposition 3.4.1 The parabolic subgroup $\mathbf{P}_{I}=\mathbf{B} W_{I} \mathbf{B}$ (see 3.2.3) has a Levi decomposition $\mathbf{P}_{I}=R_{u}\left(\mathbf{P}_{I}\right) \rtimes \mathbf{L}_{I}$ where $R_{u}\left(\mathbf{P}_{I}\right)=\mathbf{U}_{\Phi^{+}-\Phi_{I}}$ and $\mathbf{L}_{I}=$ $\left\langle\mathbf{T},\left\{\mathbf{U}_{\alpha}\right\}_{\alpha \in \Phi_{I}}\right\rangle$ is reductive. We have $\mathbf{P}_{I}=N_{\mathbf{G}}\left(R_{u}\left(\mathbf{P}_{I}\right)\right)$.

Proof The set $\Psi=\Phi^{+} \cup \Phi_{I}$ is quasi-closed since it is closed by Proposition 3.3.8. The proposition is then a consequence of 3.3.12 if we show that $\mathbf{P}_{I}=\mathbf{G}_{\Psi}$. We have $\mathbf{P}_{I} \supset \mathbf{G}_{\Psi}$ since $\mathbf{P}_{I} \supset \mathbf{U}_{\alpha}$ for $\alpha \in \Phi^{+}$since $\mathbf{P}_{I} \supset \mathbf{B}$, and by 3.2.7 $\mathbf{P}_{I}$ contains all $\mathbf{U}_{\alpha}$ for $\alpha \in \Phi^{-}$which change sign by some element of $W_{I}$, thus contains all $\mathbf{U}_{\alpha}$ for $\alpha \in \Phi_{I}^{-}$. Conversely $\mathbf{G}_{\Psi}$ contains $\mathbf{U}_{\alpha}$ and $\mathbf{U}_{-\alpha}$ for all $\alpha \in \Pi_{I}$, hence $\mathbf{G}_{\Psi}$ contains a representative of $s_{\alpha}$ in $\left\langle\mathbf{U}_{\alpha}, \mathbf{U}_{-\alpha}\right\rangle$ hence $\mathbf{G}_{\Psi}$ contains $W_{I}$, thus contains $\mathbf{P}_{I}$.

Finally, $N_{\mathbf{G}}\left(R_{u}\left(\mathbf{P}_{I}\right)\right)$ contains $\mathbf{P}_{I}$ thus $\mathbf{B}$, thus is some parabolic subgroup $\mathbf{P}_{J}$. If $J \supsetneq I$ we have $R_{u}\left(\mathbf{P}_{J}\right)=\mathbf{U}_{\Phi^{+}-\Phi_{J}} \subsetneq R_{u}\left(\mathbf{P}_{I}\right)$ which contradicts that $\mathbf{P}_{J}$ normalises $R_{u}\left(\mathbf{P}_{I}\right)$ since $R_{u}\left(\mathbf{P}_{J}\right)$ is the largest normal connected unipotent subgroup of $\mathbf{P}_{J}$.

We will say that $\mathbf{P}_{I}$ (resp. $\mathbf{L}_{I}$ ) is a standard parabolic subgroup (resp. Levi subgroup) of $\mathbf{G}$.

Proposition 3.4.2 Let $\mathbf{P}$ be a parabolic subgroup of $\mathbf{G}$ containing $\mathbf{T}$.

(i) There is a unique Levi subgroup of $\mathbf{P}$ containing $\mathbf{T}$.

(ii) Two Levi subgroups of $\mathbf{P}$ are conjugate by a unique element of $R_{u}(\mathbf{P})$. 
Proof The existence of a Levi subgroup containing $\mathbf{T}$ results from 3.4.1, since $\mathbf{P}$ is conjugate to some $\mathbf{P}_{I}$ and all maximal tori of $\mathbf{P}_{I}$ are conjugate in $\mathbf{P}_{I}$. Conversely, we may assume $\mathbf{P}=\mathbf{P}_{I}$; any Levi subgroup of $\mathbf{P}_{I}$ containing $\mathbf{T}$ is a $\mathbf{G}_{\Psi}$ for some $\Psi \subset \Phi^{+} \cup \Phi_{I}$ by Proposition 3.3.10. Since any $\mathbf{U}_{\alpha}$ where $\alpha \in \Phi^{+}-\Phi_{I}$ is in $R_{u}\left(\mathbf{P}_{I}\right)$, we must have $\Psi \subset \Phi_{I}$, thus $\mathbf{L} \subset \mathbf{L}_{I}$, thus there must be equality.

Two Levi subgroups $\mathbf{L}, \mathbf{L}^{\prime}$ of $\mathbf{P}$ are conjugate in $\mathbf{P}$, since by (i) an element which conjugates a maximal torus $\mathbf{T}$ of $\mathbf{L}$ into $\mathbf{L}^{\prime}$ conjugates $\mathbf{L}$ to $\mathbf{L}^{\prime}$. Modulo $\mathbf{L}$, we can choose the conjugating element $u$ in $R_{u}(\mathbf{P})$. The unicity of $u$ is equivalent to $R_{u}(\mathbf{P}) \cap N_{\mathbf{P}}(\mathbf{L})=1$. Assume $u \in R_{u}(\mathbf{P}) \cap N_{\mathbf{P}}(\mathbf{L})$; then for any $l \in \mathbf{L}$ we have $[u, l] \in R_{u}(\mathbf{P}) \cap \mathbf{L}=1$, thus $u \in C_{\mathbf{P}}(\mathbf{L})$; but $C_{\mathbf{P}}(\mathbf{L}) \subset C_{\mathbf{G}}(\mathbf{T})=\mathbf{T}$ thus $u=1$.

Proposition 3.4.3 The G-conjugacy classes of Levi subgroups of parabolic subgroups of $\mathbf{G}$ are in bijection with the $W$-orbits of subsets of $S$, which are themselves in bijection with the $W$-conjugacy classes of parabolic subgroups of $W$.

Proof Since all parabolic subgroups are conjugate to a $\mathbf{P}_{I}$, we may assume that we consider a Levi subgroup of some $\mathbf{P}_{I}$. Since by 3.4.2 such a Levi subgroup is $\mathbf{G}$-conjugate to $\mathbf{L}_{I}$, the question becomes that of finding when $\mathbf{L}_{J}$ is G-conjugate to $\mathbf{L}_{I}$ for two subsets $I$ and $J$ of $S$. If $\mathbf{L}_{J}={ }^{g} \mathbf{L}_{I}$ for some $g \in \mathbf{G}$, then, since ${ }^{g^{-1}} \mathbf{T}$ and $\mathbf{T}$ are two maximal tori of $\mathbf{L}_{I}$, there exists $l \in \mathbf{L}_{I}$ such that ${ }^{g^{-1}} \mathbf{T}={ }^{l} \mathbf{T}$ and $g l \in N_{\mathbf{G}}(\mathbf{T})$ also conjugates $\mathbf{L}_{I}$ to $\mathbf{L}_{J}$; so the $\mathbf{G}$-conjugacy classes of $\mathbf{L}_{I}$ are the same as the $W(\mathbf{T})$-conjugacy classes. Since $\mathbf{L}_{I}=\mathbf{G}_{\Phi_{I}}$, the element $w \in W$ conjugates $\mathbf{L}_{I}$ to $\mathbf{L}_{J}$ if and only if ${ }^{w} \Phi_{I}=\Phi_{J}$. Since any two bases of $\Phi_{I}$ are conjugate by an element of $W_{I}$ (see 2.2.6), we may assume that ${ }^{w} I=J$ whence the first part of the statement. To see the second part it is enough to see that if $w \in N_{W}\left(W_{I}\right)$ then ${ }^{w} \Phi_{I}=\Phi_{I}$. This results from Lemma 3.3.7.

The proof above shows that $N_{\mathbf{G}}\left(\mathbf{L}_{I}\right) / \mathbf{L}_{I}$ is isomorphic to $N_{W}\left(W_{I}\right) / W_{I}$.

Proposition 3.4.4 Let $\mathbf{L}$ be a Levi subgroup of a parabolic subgroup $\mathbf{P}$. Then $R(\mathbf{P})=R_{u}(\mathbf{P}) \rtimes R(\mathbf{L})$.

Proof The quotient $\mathbf{P} /\left(R(\mathbf{L}) R_{u}(\mathbf{P})\right)$ is isomorphic to $\mathbf{L} / R(\mathbf{L})$, so is semisimple. So $R(\mathbf{P}) \subset R(\mathbf{L}) R_{u}(\mathbf{P})$. But $R(\mathbf{L}) R_{u}(\mathbf{P})$ is connected, solvable and normal in $\mathbf{P}$ as the inverse image of a normal subgroup of the quotient $\mathbf{P} / R_{u}(\mathbf{P}) \simeq$ $\mathbf{L}$, whence the reverse inclusion.

We will now characterise parabolic subgroups in terms of roots.

Proposition 3.4.5 A closed subgroup $\mathbf{P}$ of $\mathbf{G}$ containing $\mathbf{T}$ is parabolic if and only if $\mathbf{P}=\mathbf{G}_{\Psi}$ for some parabolic subset $\Psi$. 
Proof We have seen that a parabolic subset $\Psi$ is conjugate under $W$ to $\Phi^{+} \cup \Phi_{I}$; thus $\mathbf{G}_{\Psi}$ is conjugate under $W$ to $\mathbf{P}_{I}$. Conversely, assume that $\mathbf{P}$ is a parabolic subgroup. It contains a Borel subgroup containing $\mathbf{T}$, thus up to conjugacy by $W$ it contains $\mathbf{B}$ (see Proposition 2.3.3), thus is of the form $\mathbf{P}_{I}$.

We now give an important property of Levi subgroups.

Proposition 3.4.6 Let $\mathbf{L}$ be a Levi subgroup of a parabolic subgroup of $\mathbf{G}$; then $\mathbf{L}=C_{\mathbf{G}}\left(Z(\mathbf{L})^{0}\right)$.

Proof We may assume that $\mathbf{L}=\mathbf{L}_{I}$. Then by 2.3.4(i) the group $Z(\mathbf{L})$ is the intersection of the kernels of the roots in $\Phi_{I}$. The group $C_{\mathbf{G}}\left(Z(\mathbf{L})^{0}\right)$ is connected as it is the centraliser of a torus $-Z(\mathbf{L})^{0}$ is diagonalisable by 1.2.1(ii) and is a torus by 1.2.3(i) thus 1.3.3(iii) applies. It is normalised by $\mathbf{T}$ because it contains $\mathbf{T}$, hence by 2.3.1(iv) it is generated by $\mathbf{T}$ and the $\mathbf{U}_{\alpha}$ it contains. If $\mathbf{U}_{\alpha} \subset C_{\mathbf{G}}\left(Z(\mathbf{L})^{0}\right)$ then $\alpha$ is trivial on $\left(\bigcap_{\alpha \in \Phi_{I}} \operatorname{Ker} \alpha\right)^{0}$. This identity component has finite index in $\bigcap_{\alpha \in \Phi_{I}} \operatorname{Ker} \alpha$, hence some multiple $n \alpha$ of $\alpha$ is trivial on $\bigcap_{\alpha \in \Phi_{I}} \operatorname{Ker} \alpha$. With the notation of 1.2.12, this can be rewritten as $n \alpha \in\left(\left\langle\Phi_{I}\right\rangle_{\mathbf{T}}^{\perp}\right)_{X(\mathbf{T})}^{\perp}$. But $\left(\left\langle\Phi_{I}\right\rangle_{\mathbf{T}}^{\perp}\right)_{X(\mathbf{T})}^{\perp} /\left\langle\Phi_{I}\right\rangle$ is a torsion group (see 1.2.13). This implies that $\alpha \in\left\langle\Phi_{I}\right\rangle \otimes \mathbb{Q}$, which in turn yields $\alpha \in \Phi_{I}$ by the definition of $\Phi_{I}$. This proves that $C_{\mathbf{G}}\left(Z(\mathbf{L})^{0}\right) \subset \mathbf{L}$. The reverse inclusion is obvious.

The next proposition is a kind of converse.

Proposition 3.4.7 For any torus $\mathbf{S}$, the group $C_{\mathbf{G}}(\mathbf{S})$ is a Levi subgroup of some parabolic subgroup of $\mathbf{G}$.

Proof Let $\mathbf{T}$ be a maximal torus containing $\mathbf{S}$. As the group $C_{\mathbf{G}}(\mathbf{S})$ is connected by 1.3.3(iii) and contains $\mathbf{T}$, by 2.3.1(iv) we have $C_{\mathbf{G}}(\mathbf{S})=\left\langle\mathbf{T}, \mathbf{U}_{\alpha}\right| \mathbf{U}_{\alpha} \subset$ $\left.C_{\mathbf{G}}(\mathbf{S})\right\rangle$. As $\mathbf{S}$ acts by $\alpha$ on $\mathbf{U}_{\alpha}$ (see 2.3.1(i)), we have

$$
\left.\mathbf{U}_{\alpha} \subset C_{\mathbf{G}}(\mathbf{S}) \Leftrightarrow \alpha\right|_{\mathbf{S}}=0,
$$

where 0 is the trivial element of $X(\mathbf{S})$. Let us choose a total order on $X(\mathbf{S})$; that is, a structure of ordered $\mathbb{Z}$-module. As $X(\mathbf{S})$ is a quotient of $X(\mathbf{T})$ (see 1.2.4) there exists a total order on $X(\mathbf{T})$ compatible with the chosen order on $X(\mathbf{S})$; that is, such that for $x \in X(\mathbf{T})$ we have $x \geq\left. 0 \Rightarrow x\right|_{\mathbf{S}} \geq 0$. This implies that the set $\Psi=\left\{\alpha \in \Phi \mid \alpha>0\right.$ or $\left.\left.\alpha\right|_{\mathbf{S}}=0\right\}$ is also equal to $\left\{\alpha \in \Phi|\alpha|_{\mathbf{S}} \geq 0\right\}$. This last definition implies that $\Psi$ is closed, so (see 3.3.13 and 3.3.10) $\Psi$ is also the set of $\alpha$ such that $\mathbf{U}_{\alpha} \subset \mathbf{G} \Psi$. Since $\Psi$ is parabolic, it follows then from 3.4.5 that $\mathbf{G}_{\Psi}$ is a parabolic subgroup, of which $C_{\mathbf{G}}(\mathbf{S})$ is a Levi complement.

We now study the intersection of two parabolic subgroups. First note that by 3.1.4 the intersection of two parabolic subgroups always contains some maximal torus of $\mathbf{G}$. 
Proposition 3.4.8 Let $\mathbf{P}$ and $\mathbf{P}^{\prime}$ be two parabolic subgroups of $\mathbf{G}$ and let $\mathbf{L}$ and $\mathbf{L}^{\prime}$ be respective Levi subgroups of $\mathbf{P}$ and $\mathbf{P}^{\prime}$ containing the same maximal torus $\mathbf{T}$ of $\mathbf{G}$. Let $\mathbf{U}=R_{u}(\mathbf{P})$ and $\mathbf{U}^{\prime}=R_{u}\left(\mathbf{P}^{\prime}\right)$. Then

(i) The group $\left(\mathbf{P} \cap \mathbf{P}^{\prime}\right) . \mathbf{U}$ is a parabolic subgroup of $\mathbf{G}$ which has the same intersection as $\mathbf{P}^{\prime}$ with $\mathbf{L}$, and it has $\mathbf{L} \cap \mathbf{L}^{\prime}$ as a Levi subgroup.

(ii) The group $\mathbf{P} \cap \mathbf{P}^{\prime}$ is connected, as well as $\mathbf{L} \cap \mathbf{L}^{\prime}$ and we have the Levi decomposition

$$
\mathbf{P} \cap \mathbf{P}^{\prime}=\left(\left(\mathbf{L} \cap \mathbf{U}^{\prime}\right) .\left(\mathbf{L}^{\prime} \cap \mathbf{U}\right) .\left(\mathbf{U} \cap \mathbf{U}^{\prime}\right)\right) \rtimes\left(\mathbf{L} \cap \mathbf{L}^{\prime}\right)
$$

where the right-hand side is a direct product of varieties - the decomposition of an element of $\mathbf{P} \cap \mathbf{P}^{\prime}$ as a product of four terms is unique. On the right-hand side the last factor is a Levi subgroup of $\mathbf{P} \cap \mathbf{P}^{\prime}$ and the first 3 factors form a decomposition of $R_{u}\left(\mathbf{P} \cap \mathbf{P}^{\prime}\right)$.

Proof Let $\Phi$ be the roots of $\mathbf{G}$ with respect to $\mathbf{T}$ and define subsets $\Psi, \Psi^{\prime} \subset \Phi$ by $\mathbf{P}=\mathbf{G}_{\Psi}$ and $\mathbf{P}^{\prime}=\mathbf{G}_{\Psi^{\prime \prime}}$.

Let us show first that for any $\alpha \in \Phi$, either $\mathbf{U}_{\alpha}$ or $\mathbf{U}_{-\alpha}$ is in the group $\left(\mathbf{P} \cap \mathbf{P}^{\prime}\right) \cdot \mathbf{U}$ (it is a group since $\mathbf{P}$ normalises $\mathbf{U}$ ). By the remarks before 3.3.11 and by 3.3.12 we have $\left(\mathbf{P} \cap \mathbf{P}^{\prime}\right)^{0} \cdot \mathbf{U}=\mathbf{G}_{\Psi_{\cap} \Psi^{\prime}} \cdot \mathbf{G}_{\Psi_{u}}^{*}$, with the notation of 3.3.12. If neither $\alpha$ nor $-\alpha$ is in $\Psi_{u}$, they are both in $\Psi$ in which case since one of them is in $\Psi^{\prime}$, one of them is in $\Psi \cap \Psi^{\prime}$. Hence $\left(\Psi \cap \Psi^{\prime}\right) \cup \Psi_{u}$ is a parabolic set; indeed, this set is closed as the sum of an element of $\Psi$ and an element of $\Psi_{u}$ which is a root is in $\Psi_{u}$ - see the beginning of the proof of 3.3.13. Proposition 3.4.5 then shows that $\left(\mathbf{P} \cap \mathbf{P}^{\prime}\right)^{0}$. $\mathbf{U}$ is a parabolic subgroup of $\mathbf{G}$, equal to $\mathbf{G}_{\left(\Psi \cap \Psi^{\prime}\right) \cup \Psi_{u}}$. Then $\left(\mathbf{P} \cap \mathbf{P}^{\prime}\right) \mathbf{U}$, containing a parabolic subgroup is connected, hence equal to $\left(\mathbf{P} \cap \mathbf{P}^{\prime}\right)^{0} . \mathbf{U}$.

Now $\left(\mathbf{P} \cap \mathbf{P}^{\prime}\right) \cdot \mathbf{U}=\left(\mathbf{P} \cap \mathbf{P}^{\prime}\right) \cdot \mathbf{G}_{\Psi_{u}-\Psi^{\prime}}^{*}$ since $\Psi^{\prime} \cap \Psi_{u} \subset \Psi \cap \Psi^{\prime}$. The set $\Psi_{u}-\Psi^{\prime}$ is closed as the intersection of the closed subsets $\Psi_{u}$ and the complement $-\Psi_{u}^{\prime}$ of $\Psi^{\prime}$, hence the product $\left(\mathbf{P} \cap \mathbf{P}^{\prime}\right) . \mathbf{G}_{\Psi_{u}-\Psi^{\prime}}^{*}$ is a direct product of varieties as the intersection is a unipotent subgroup normalised by $\mathbf{T}$ containing no $\mathbf{U}_{\alpha}$, see 2.3.1(v). As the product is connected, each term is. Thus $\mathbf{P} \cap \mathbf{P}^{\prime}$ is connected equal to $\mathbf{G}_{\Psi \cap \Psi^{\prime}}$. The groups $\left(\mathbf{P} \cap \mathbf{P}^{\prime}\right) . \mathbf{U}$ and $\mathbf{P} \cap \mathbf{P}^{\prime}$ have both $\left(\mathbf{L} \cap \mathbf{L}^{\prime}\right)^{0}$ as a Levi subgroup since $\left(\left(\Psi \cap \Psi^{\prime}\right) \cup \Psi_{u}\right)_{s}=\left(\Psi \cap \Psi^{\prime}\right)_{s}=\Psi_{s} \cap \Psi_{s}^{\prime}$ - indeed if $\alpha \in \Psi_{u}$ then $-\alpha \notin \Psi$ thus $-\alpha \notin\left(\Psi \cap \Psi^{\prime}\right) \cup \Psi_{u}$.

The decomposition $\Psi \cap \Psi^{\prime}=\left(\Psi_{s} \cap \Psi_{s}^{\prime}\right) \amalg\left(\Psi_{s} \cap \Psi_{u}^{\prime}\right) \amalg\left(\Psi_{u} \cap \Psi_{s}^{\prime}\right) \amalg\left(\Psi_{u} \cap \Psi_{u}^{\prime}\right)$ shows that $\mathbf{P} \cap \mathbf{P}^{\prime}=\left\langle\mathbf{L} \cap \mathbf{L}^{\prime}, \mathbf{L} \cap \mathbf{U}^{\prime}, \mathbf{L}^{\prime} \cap \mathbf{U}, \mathbf{U} \cap \mathbf{U}^{\prime}\right\rangle$. Using that $\mathbf{U} \cap \mathbf{U}^{\prime}$ is normal in $\mathbf{P} \cap \mathbf{P}^{\prime}$, then that $\mathbf{L} \cap \mathbf{L}^{\prime}$ normalises $\mathbf{L} \cap \mathbf{U}^{\prime}$ and $\mathbf{L}^{\prime} \cap \mathbf{U}$, we get

$$
\mathbf{P} \cap \mathbf{P}^{\prime}=\left(\mathbf{L} \cap \mathbf{L}^{\prime}\right) .\left\langle\mathbf{L} \cap \mathbf{U}^{\prime}, \mathbf{L}^{\prime} \cap \mathbf{U}\right\rangle .\left(\mathbf{U} \cap \mathbf{U}^{\prime}\right) .
$$


Further, the commutator of an element of $\mathbf{L} \cap \mathbf{U}^{\prime}$ with an element of $\mathbf{L}^{\prime} \cap \mathbf{U}$ is in $\mathbf{U} \cap \mathbf{U}^{\prime}$, thus

$$
\mathbf{P} \cap \mathbf{P}^{\prime}=\left(\mathbf{L} \cap \mathbf{L}^{\prime}\right) .\left(\mathbf{L} \cap \mathbf{U}^{\prime}\right) .\left(\mathbf{L}^{\prime} \cap \mathbf{U}\right) .\left(\mathbf{U} \cap \mathbf{U}^{\prime}\right),
$$

Write now $x=l u^{\prime} u v \in \mathbf{P} \cap \mathbf{P}^{\prime}$, where $l \in \mathbf{L} \cap \mathbf{L}^{\prime}, u^{\prime} \in \mathbf{L} \cap \mathbf{U}^{\prime}, u \in \mathbf{L}^{\prime} \cap \mathbf{U}$, $v \in \mathbf{U} \cap \mathbf{U}^{\prime}$. Then $l u^{\prime}$ is the image of $x$ by the projection $\mathbf{P} \rightarrow \mathbf{L}$ and $l$ (resp. $u$ ) is the image of $l u^{\prime}$ (resp. $u v$ ) by the morphism $\mathbf{P}^{\prime} \rightarrow \mathbf{L}^{\prime}$. Thus the decomposition of $x$ is unique, and the product map $\left(\mathbf{L} \cap \mathbf{L}^{\prime}\right) \times\left(\mathbf{L} \cap \mathbf{U}^{\prime}\right) \times\left(\mathbf{L}^{\prime} \cap \mathbf{U}\right) \times\left(\mathbf{U} \cap \mathbf{U}^{\prime}\right) \rightarrow$ $\mathbf{P} \cap \mathbf{P}^{\prime}$ is an isomorphism of varieties; the four terms are connected since the product is. In particular $\mathbf{L} \cap \mathbf{L}^{\prime}$ is connected.

\section{Proposition 3.4.9}

(i) Let $\mathbf{P}$ and $\mathbf{P}^{\prime}$ be two parabolic subgroups of $\mathbf{G}$ such that $\mathbf{P}^{\prime} \subset \mathbf{P}$, then $R_{u}\left(\mathbf{P}^{\prime}\right) \supset R_{u}(\mathbf{P})$ and for any Levi subgroup $\mathbf{L}^{\prime}$ of $\mathbf{P}^{\prime}$, there exists a unique Levi subgroup $\mathbf{L}$ of $\mathbf{P}$ such that $\mathbf{L} \supset \mathbf{L}$.

(ii) Let $\mathbf{L}$ be a Levi subgroup of a parabolic subgroup $\mathbf{P}$ of $\mathbf{G}$ and $\mathbf{L}^{\prime}$ be a closed subgroup of $\mathbf{L}$. Then the following are equivalent:

(a) $\mathbf{L}^{\prime}$ is a Levi subgroup of a parabolic subgroup of $\mathbf{L}$.

(b) $\mathbf{L}^{\prime}$ is a Levi subgroup of a parabolic subgroup of $\mathbf{G}$.

Proof Let us prove (i); given a maximal torus $\mathbf{T}$ of $\mathbf{L}^{\prime}$ there is by 3.4.2(i) a unique Levi subgroup $\mathbf{L}$ of $\mathbf{P}$ containing $\mathbf{T}$. Then by 3.4.8(ii) the group $\mathbf{L}^{\prime} \cap \mathbf{L}$ is a Levi subgroup of $\mathbf{P}^{\prime}=\mathbf{P} \cap \mathbf{P}^{\prime}$ thus $\mathbf{L} \cap \mathbf{L}^{\prime}=\mathbf{L}^{\prime}$. Also $R_{u}(\mathbf{P})$ is contained in all Borel subgroups of $\mathbf{P}$, thus in $\mathbf{P}^{\prime}$, whence $R_{u}(\mathbf{P}) \subset R_{u}\left(\mathbf{P}^{\prime}\right)$.

Let us show (ii); if $\mathbf{L}^{\prime}$ is a Levi subgroup of the parabolic subgroup $\mathbf{P}_{\mathbf{L}}$ of $\mathbf{L}$, then $\mathbf{P}_{\mathbf{L}} R_{u}(\mathbf{P})$ is a parabolic subgroup of $\mathbf{G}$; indeed it is a group since $\mathbf{L}$, thus $\mathbf{P}_{\mathbf{L}}$, normalises $R_{u}(\mathbf{P})$ and it clearly contains either $\mathbf{U}_{\alpha}$ or $\mathbf{U}_{-\alpha}$ for any $\alpha \in$ $\Phi$. Thus $\mathbf{L}^{\prime}$ is a Levi subgroup of $\mathbf{P}_{\mathbf{L}} R_{u}(\mathbf{P})$, since $R_{u}\left(\mathbf{P}_{\mathbf{L}}\right) \cdot R_{u}(\mathbf{P})$ is unipotent normal in $\mathbf{P}_{\mathbf{L}} \cdot R_{u}(\mathbf{P})$. We have shown that (a) implies (b).

Conversely, let $\mathbf{P}^{\prime}$ be a parabolic subgroup of $\mathbf{G}$ with $\mathbf{L}^{\prime}$ as a Levi subgroup. By 3.4.8(ii) we have $\mathbf{P} \cap \mathbf{P}^{\prime}=\mathbf{L}^{\prime}$. $\left(\mathbf{L} \cap \mathbf{U}^{\prime}\right)$. $\left(\mathbf{U} \cap \mathbf{U}^{\prime}\right)$ thus $\left(\mathbf{L} \cap \mathbf{U}^{\prime}\right) \rtimes \mathbf{L}^{\prime}$ is a Levi decomposition of $\mathbf{L} \cap \mathbf{P}^{\prime}$, and this last group is a parabolic subgroup of $\mathbf{L}$ by 3.4 .5 .

When $\mathbf{L}$ is a Levi subgroup of some parabolic subgroup of $\mathbf{G}$ we will say (improperly) " $\mathbf{L}$ is a Levi subgroup of $\mathbf{G}$ " which is justified by statement (ii) of 3.4.9.

Proposition 3.4.10 Let $\mathbf{H}$ be a closed connected reductive subgroup of $\mathbf{G}$ of maximal rank. Then: 
(i) The Borel subgroups of $\mathbf{H}$ are the $\mathbf{B} \cap \mathbf{H}$ where $\mathbf{B}$ is a Borel subgroup of $\mathbf{G}$ containing a maximal torus of $\mathbf{H}$.

(ii) The parabolic subgroups of $\mathbf{H}$ are the $\mathbf{P} \cap \mathbf{H}$, where $\mathbf{P}$ is a parabolic subgroup of $\mathbf{G}$ containing a maximal torus of $\mathbf{H}$.

(iii) If $\mathbf{P}$ is a parabolic subgroup of $\mathbf{G}$ containing a maximal torus of $\mathbf{H}$, the Levi subgroups of $\mathbf{P} \cap \mathbf{H}$ are the $\mathbf{L} \cap \mathbf{H}$ where $\mathbf{L}$ is a Levi subgroup of $\mathbf{P}$ containing a maximal torus of $\mathbf{H}$.

Proof Let $\mathbf{T}$ be a maximal torus of $\mathbf{H}$; by assumption, it is also a maximal torus of $\mathbf{G}$. Let $\mathbf{B}$ be a Borel subgroup of $\mathbf{G}$ containing $\mathbf{T}$, and let $\mathbf{B}=\mathbf{U}$.T be the corresponding semi-direct product decomposition. The Borel subgroup $\mathbf{B}$ defines an order on the root system $\Phi\left(\right.$ resp. $\left.\Phi_{\mathbf{H}}\right)$ of $\mathbf{G}$ (resp. $\left.\mathbf{H}\right)$ with respect to $\mathbf{T}$. The group $\mathbf{U} \cap \mathbf{H}$ is normalised by $\mathbf{T}$, so is connected and equal to the product of the $\mathbf{U}_{\alpha}$ it contains, that is those $\mathbf{U}_{\alpha}$ such that $\alpha$ is positive and in $\Phi_{\mathbf{H}}$, so $(\mathbf{U} \cap \mathbf{H}) . \mathbf{T}=\mathbf{B} \cap \mathbf{H}$ is a Borel subgroup of $\mathbf{H}$. This gives (i) since all Borel subgroups of $\mathbf{H}$ are conjugate under $\mathbf{H}$.

Let us prove (ii). If $\mathbf{P}$ is a parabolic subgroup of $\mathbf{G}$ containing $\mathbf{T}$, it contains a Borel subgroup $\mathbf{B}$ containing $\mathbf{T}$, so its intersection with $\mathbf{H}$ contains the Borel subgroup $\mathbf{B} \cap \mathbf{H}$ of $\mathbf{H}$ and thus is a parabolic subgroup. Conversely, let $\mathbf{Q}$ be a parabolic subgroup of $\mathbf{H}$ containing $\mathbf{T}$, and let $x$ be a vector of $X(\mathbf{T}) \otimes \mathbb{Q}$ defining $\mathbf{Q}$ as in 3.3.8(ii). Then $x$ defines a parabolic subgroup $\mathbf{P}$ of $\mathbf{G}$. It remains to show that $\mathbf{P} \cap \mathbf{H}=\mathbf{Q}$. The group $\mathbf{P} \cap \mathbf{H}$ is a parabolic subgroup of $\mathbf{H}$ by the first part. It is generated by $\mathbf{T}$ and the $\mathbf{U}_{\alpha}$ it contains. But $\mathbf{U}_{\alpha} \subset \mathbf{P} \cap \mathbf{H}$ if and only if $\alpha \in \Phi_{\mathbf{H}}$ and $\langle\alpha, x\rangle \geq 0$; that is, if and only if $\mathbf{U}_{\alpha} \subset \mathbf{Q}$ by definition of $x$.

Similarly, the Levi subgroup of $\mathbf{Q}$ containing $\mathbf{T}$ is the intersection of the Levi subgroup of $\mathbf{P}$ containing $\mathbf{T}$ with $\mathbf{H}$, as it is generated by $\mathbf{T}$ and the $\mathbf{U}_{\alpha}$ with $\alpha \in \Phi_{\mathbf{H}}$ orthogonal to $x$, whence (iii).

\subsection{Centralisers of Semi-Simple Elements}

Proposition 3.5.1 Let $s \in \mathbf{G}$ be a semi-simple element, and let $\mathbf{T}$ be a maximal torus containing $s$ and $\Phi$ be the set of roots of $\mathbf{G}$ relative to $\mathbf{T}$; then

(i) The identity component $C_{\mathbf{G}}(s)^{0}$ is generated by $\mathbf{T}$ and the $\mathbf{U}_{\alpha}$ for $\alpha \in \Phi$ such that $\alpha(s)=1$. It is a connected reductive subgroup of $\mathbf{G}$ of maximal rank.

(ii) $C_{\mathbf{G}}(s)$ is generated by $C_{\mathbf{G}}(s)^{0}$ and the elements $n \in N_{\mathbf{G}}(\mathbf{T})$ such that ${ }^{n} s=s$.

Proof (i) is an immediate consequence of 2.3.1(iv), and of the fact that the corresponding set of $\alpha$ is closed and symmetric - see 3.3.12 and 3.3.13. 
Let us prove (ii). Let $\mathbf{B}=\mathbf{U} \rtimes \mathbf{T}$ be the Levi decomposition of a Borel subgroup of $\mathbf{G}$ and let $g \in C_{\mathbf{G}}(s)$; by Lemma 3.2.7 the element $g$ has a unique decomposition $g=u n v$ with $n \in N_{\mathbf{G}}(\mathbf{T}), u \in \mathbf{U}$ and $v \in \mathbf{U}_{w}$ where $w$ is the image of $n$ in $W(\mathbf{T})$. As $s$ normalises $\mathbf{U}, \mathbf{U}_{w}$ and $N_{\mathbf{G}}(\mathbf{T})$, this decomposition is invariant under conjugation by $s$, so each of $u, n$ and $v$ also centralises $s$. Writing again a unique decomposition of the form $u=\prod_{\alpha>0} u_{\alpha}$ we see that the $\alpha$ must satisfy $\alpha(s)=1$ so $u \in C_{\mathbf{G}}(s)^{0}$, and the same argument applies to $v$. Thus we get (ii).

Remark 3.5.2 The Weyl group $W^{0}(s)$ of $C_{\mathbf{G}}(s)^{0}$ is thus the group generated by the reflections $s_{\alpha}$ for which $\alpha(s)=1$. It is a normal subgroup of the Weyl group of $C_{\mathbf{G}}(s)$ which is $W(s)=\left\{w \in W(\mathbf{T}) \mid{ }^{w} s=s\right\}$. The quotient $W(s) / W^{0}(s)$ is isomorphic to the quotient $C_{\mathbf{G}}(s) / C_{\mathbf{G}}(s)^{0}$.

Proposition 3.5.3 If $x=$ su is the Jordan decomposition of an element of $\mathbf{G}$, where $s$ is semi-simple and $u$ unipotent, then $x \in C_{\mathbf{G}}(s)^{0}$.

Proof Let $\mathbf{B}=\mathbf{U} \rtimes \mathbf{T}$ be a Levi decomposition of a Borel subgroup containing $x$, where $\mathbf{T}$ is a maximal torus of $\mathbf{B}$ containing $s$, and write $u=\prod u_{\alpha}$ (with $u_{\alpha} \in$ $\mathbf{U}_{\alpha}$ where $\left.\mathbf{U}=\prod \mathbf{U}_{\alpha}\right)$. Then for any root $\alpha$ such that $u_{\alpha} \neq 1$, we have $\alpha(s)=1$ which implies that $\mathbf{U}_{\alpha} \subset C_{\mathbf{G}}(s)^{0}$, whence the result as $s \in \mathbf{T} \subset C_{\mathbf{G}}(s)^{0}$.

Examples 3.5.4

(i) All centralisers in $\mathbf{G L}_{n}$ are connected. Indeed, the centraliser in the variety of all matrices is an affine space, thus its intersection with $\mathbf{G L}_{n}$ is an open subspace of an affine space, which is always connected.

(ii) In the group $\mathbf{S} \mathbf{L}_{n}$, centralisers of semi-simple elements are connected. Indeed such an element is conjugate to an element $s=\operatorname{diag}\left(t_{1}, \ldots, t_{n}\right)$ of the torus $\mathbf{T}$ of diagonal matrices where we may assume, in addition, that equal $t_{i}$ are grouped in consecutive blocks, thereby defining a partition $\pi$ of $n$. The elements of $W(\mathbf{T})$ (permutation matrices) which centralise $s$ are products of generating reflections $s_{\alpha}$ which centralise $s$; that is, $W(s)=W^{0}(s)$ showing that the centraliser of $s$ is connected.

(iii) We finish with an example of a semi-simple element whose centraliser is not connected. Let $s \in \mathbf{P G L}_{2}$ be the image of $\left(\begin{array}{cc}1 & 0 \\ 0 & -1\end{array}\right)$; in characteristic different from 2, $C_{\mathbf{P G L}_{2}}(s)$ has two connected components, consisting respectively of the images of the matrices of the form $\left(\begin{array}{ll}a & 0 \\ 0 & b\end{array}\right)$ and of the form $\left(\begin{array}{ll}0 & a \\ b & 0\end{array}\right)$. 


\section{Notes}

A classic reference about $(B, N)$-pairs is Bourbaki (1968, Chapter IV). A detailed study of closed and quasi-closed subsets and reductive and parabolic subgroups is in Borel and Tits (1965). A detailed study of the centralisers of semi-simple elements can be found in, for example, Deriziotis (1984). 